\title{
CINÉTICA DE TRANSFERÊNCIA DE MASSA DE MELÃO DESIDRATADO OSMOTICAMENTE EM SOLUÇÕES DE SACAROSE E MALTOSE
}

\author{
C.C. FERRARI', L.K. RODRIGUES', R.V. TONON', M.D. HUBINGER
}

\section{RESUMO}

Cubos de melão de $20 \mathrm{~mm}$ da variedade Cucumis melo inodorus, cultivar Gold Mine foram desidratados osmoticamente com o objetivo de se estudar a cinética de transferência de massa durante o processo. As amostras foram imersas em soluções hipertônicas de sacarose ou maltose nas concentrações de 40 a $60^{\circ}$ Brix e a desidratação foi conduzida por 8 horas com temperatura controlada $\left(30\right.$ ou $\left.40^{\circ} \mathrm{C}\right)$ e agitação de $120 \mathrm{rpm}$. Perda de água e incorporação de sólidos na fruta foram avaliados ao longo do processo em função da temperatura de tratamento, tipo e concentração da solução. Os coeficientes de difusão para a água e os açúcares foram estimados através de um modelo empírico da literatura, baseado na equacão de Fick. Para todos os ensaios, a perda de água aumentou significativamente com a elevação da temperatura e da concentração da solução desidratante, entretanto, os ensaios com maltose promoveram uma maior taxa de saída de água da fruta e menor ganho de sólidos. O modelo empírico utilizado se ajustou adequadamente aos dados experimentais, apresentando valores do coeficiente de correlação (R ) superiores a 0,92.

Palavras-chave: desidratação osmótica; melão; difusividade efetiva; perda de água; ganho de sólidos.

\section{SUMMARY}

MASS TRANSFER KINETICS OF OSMODEHYDRATED MELON WITH SUCROSE AND MALTOSE SOLUTIONS. Melon cubes of $20 \mathrm{~mm}$ from the Cucumis melo inodorus variety, Gold Mine cultivar were osmotically dehydrated with the aim of studying the mass transfer kinetics during the process. The samples were immersed in hypertonic solution of sucrose or maltose with different concentrations $\left(40\right.$ to $\left.60^{\circ} \mathrm{Brix}\right)$ and the osmotic dehydration was carried out for eight hours under controlled temperature $\left(30^{\circ} \mathrm{C}\right.$ or $40^{\circ} \mathrm{C}$ ) and agitation $(120 \mathrm{rpm})$. Water loss and solids gain were evaluated throughout the process as a function of temperature, osmotic agent and solution concentration. The diffusion coefficients for water and sugar were estimated using an empiric model from literature based on Fick's unsteady law of diffusion. For all treatments, water removal increased at a significant level for higher temperature and solution concentrations, but the greatest water loss rate and lowest sugar uptake was verified when using maltose solutions. The empiric model showed a good fit to experimental values with a high degree of correlation $\left(R^{2}>0,92\right)$.

Keywords: osmotic dehydration; melon; effective diffusivity; water loss; solids gain.

\section{1 - INTRODUÇÃO}

O Brasil é atualmente um dos maiores produtores de melão do mundo, sendo a Região Nordeste responsável por $95 \%$ da produção nacional. O estado do Rio Grande do Norte concentra cerca de $65 \%$ da produção total brasileira, além de se destacar como um dos maiores pólos exportadores de melão do país e, em seguida, estão os estados do Ceará, Bahia e Pernambuco [1].

Apesar de ser considerado um produto de elevado valor comercial e ser apreciado por suas características sensoriais, o melão apresenta uma vida útil pós-colheita relativamente curta à temperatura ambiente, o que tem dificultado consideravelmente a sua comercialização nos mercados mais distantes dos centros de produção, contribuindo para o elevado desperdício desse fruto [15].

O melão, diferentemente de outros frutos como, maçã e banana, possui baixo teor de amido, polissacarídeo, que quando em alta concentração no fruto, contribui de forma significativa para o aumento do teor de sólidos solúveis totais durante o amadurecimento. Para isso, é ne-

Recebido para publicação em 04/02/2005. Aceito para publicação em 11/08/2005(001476).

Departamento de Engenharia de Alimentos, Faculdade de Engenharia de Alimentos, Universidade Estadual de Campinas. Caixa Postal 6121. CEP: 13083-970, Campinas-SP, Brasil. Tel: 55(19)3788-4036. E-mail: mhub@fea.unicamp.br

A quem a correspondência deve ser enviada. cessário que o fruto permaneça na planta até atingir a completa maturação para a garantia de um produto com alto teor de sólidos solúveis e, assim, boa qualidade [16]. No entanto, na tentativa de prolongar o período de vida útil pós-colheita, o melão é freqüentemente colhido antes da sua completa maturação, não alcançando o teor mínimo de sólidos solúveis totais de 8 a $10^{\circ} \mathrm{Brix}$, utilizado como guia de mercado para a aceitação do produto em muitos países [17].

Nesse contexto, a utilização do processo de desidratação osmótica pode garantir um produto final com maior teor de açúcar, o que significa uma melhora das qualidades sensoriais do melão, um aumento de vida de prateleira e características mais uniformes no produto pré-processado, devido principalmente à retirada parcial da água e ao efeito protetor do soluto utilizado.

Nas últimas décadas, estudos relacionados com a desidratação osmótica revelaram a eficiência da utilização dessa técnica como etapa preliminar ou adicional aos processos de secagem e congelamento, obtendo-se produtos de alta qualidade sensorial e nutricional e mais estáveis à contaminação microbiológica e à deterioração química [25]. A característica diferencial do processo osmótico em relação a outras técnicas de desidratação de alimentos é a possibilidade de modificar a sua formulação através da incorporação de solutos na estrutura porosa das frutas e hortaliças. Esses solutos podem ser depressores de $\mathrm{pH}$ ou de atividade de água, componentes fisiologicamente ativos, antimicrobianos, entre outros, que possam favorecer a preservação sensorial e nutricional dos produtos, 
além de formular produtos funcionais, estáveis e mais próximos aos frutos frescos [8].

Para a escolha do agente desidratante existem considerações a serem feitas, como as mudanças no valor nutritivo e nas propriedades sensoriais do produto final e o custo do processo. Diante disso, a sacarose é o soluto mais utilizado para frutas em virtude de sua eficiência, conveniência, custo e aroma desejável provocado na fruta [14]. Entretanto, outros dissacarídeos têm sido empregados no processo osmótico como a maltose, devido ao seu maior efeito protetor nas propriedades de cor, na retenção de ácido ascórbico durante a secagem de produtos previamente desidratados osmoticamente e na preservação da funcionalidade da membrana celular [9]. Além disso, a maltose apresenta peso molecular relativamente maior do que a sacarose, possibilitando uma maior taxa de perda de água e um ganho de sólidos reduzido, de modo a evitar um impacto negativo nas propriedades sensoriais do produto, por conseqüência da indesejável e elevada impregnação de sólidos [3, 12].

O efeito da concentração da solução na transferência de massa durante o processo osmótico, utilizando sacarose como agente desidratante, tem sido avaliado por diversos pesquisadores. Na desidratação osmótica de pêra [19], cenoura [21], abacaxi [26] e melão [15], os autores verificaram que o gradiente de pressão osmótica é responsável pela difusão da água do alimento para a solução, sendo que maiores concentrações de solução resultam num aumento do gradiente de pressão osmótica e, conseqüentemente, maiores taxas de perda de água são obtidas. Por outro lado, apesar das vantagens do uso da maltose no processo, estudos de cinética de desidratação osmótica empregando este dissacarídeo como agente desidratante são praticamente inexistentes.

Segundo AZUARA, BERISTAIN \& GARCIA [5], as equações utilizadas para descrever a cinética do processo osmótico são complexas e geralmente específicas para certas condições de processo e configurações geométricas, além de não predizerem o ponto de equilíbrio. Diante disso, a partir de um balanço de massa, os autores encontraram uma equação capaz de predizer a cinética de desidratação osmótica e o ponto final do equilíbrio, sem a necessidade de se chegar ao equilíbrio, utilizando apenas um curto período de processo. O modelo pode ser usado para caracterizar a desidratação osmótica de diferentes tipos de alimentos, sem restrições de geometria, e foi aplicado na determinação da cinética de transferência de massa de tomate-cereja [4], mamão [7] e maçãs [11] desidratadas osmoticamente, apresentando um ajuste satisfatório aos dados experimentais.

O objetivo do presente trabalho foi estudar a influência das variáveis tipo de açúcar, concentração e temperatura da solução desidratante na transferência de massa durante a desidratação osmótica de cubos de melão, através da determinação dos coeficientes de difusão da água e dos solutos utilizando um modelo cinético empírico da literatura baseado na equação de Fick.

\section{2 - MATERIAL E MÉTODOS}

\section{1 - Matéria-prima}

Foi utilizado melão da variedade Cucumis melo inodorus, cultivar Gold Mine fornecido pelo CEASA Campinas/SP, usando como critério de seleção: nivel de maturação, intensidade da cor e Brix $\left(9-10^{\circ}\right.$ Brix), mesmo tamanho e forma, a fim de se obter amostras relativamente homogêneas.

A caracterização da matéria-prima foi realizada através das análises de umidade, cinzas, proteínas, açúcares redutores e totais e acidez [2] e sólidos solúveis totais por refratometria, com o auxilio de um refratômetro de bancada Zeiss (West Germany). O pH foi determinado com pHmetro modelo pH300 (Analyser Comércio e Indústria Ltda.) e a atividade de água utilizando-se o equipamento Aqualab, modelo CX-2 (Decagon Devices Inc., Pullman, WA), a $25^{\circ} \mathrm{C}$, previamente calibrado com amostras padrão de soluções saturadas de $\mathrm{KCl}$ e água deionizada, sendo que foram consideradas as leituras com desvios de $\pm 0,003$.

A Tabela 1 indica a caracterização físico-química do melão da variedade Cucumis melo inodorus, cultivar Gold Mine utilizado nos experimentos. Os dados apresentados correspondem a uma média de, no mínimo, três determinações com seu desvio padrão.

TABELA 1 - Caracterização físico-química do melão in natura da variedade Cucumis melo inodorus, cultivar Gold Mine

\begin{tabular}{lc}
\hline \multicolumn{1}{c}{ Análise } & Valor Médio \pm Desvio Padrão \\
\hline Umidade (base úmida) (\%) & $90,59 \pm 1,13$ \\
Cinzas (\%) & $0,41 \pm 0,01$ \\
Proteinas (\%) & $0,42 \pm 0,02$ \\
Açúcares Totais (\%) & $8,34 \pm 0,83$ \\
Açúcares Redutores $(\%)$ & $4,81 \pm 0,51$ \\
Acidez Titulável (g 100 $\mathrm{mL}^{-1}$ ) & $0,11 \pm 0,01$ \\
Sólidos Solúveis ( ${ }^{\circ}$ Brix) & $9,50 \pm 0,83$ \\
pH & $5,53 \pm 0,09$ \\
Atividade de Água ( $\left(\mathrm{a}_{w}\right)$ & $0,982 \pm 0,002$ \\
\hline
\end{tabular}

\section{2 - Desidratação osmótica}

As soluções desidratantes foram preparadas com água destilada nas concentrações de 40 a $60^{\circ} \mathrm{Brix}$, utilizando como agente desidratante sacarose comercial (marca União) e maltose (xarope de milho de alta maltose Maltegill ${ }^{\circ}$ com D.E. $=63 \%$ e Sólidos Totais $=82 \%$ ) adquirida da Cargill do Brasil.

Os frutos foram lavados em água corrente e mergulhados por 10 minutos numa solução de Desinfetante Clorado para Frutas e Verduras - Sumaveg, da Diversey Lever, na concentração de $0,66 \%(\mathrm{p} / \mathrm{v})$. Após a lavagem, os melões foram descascados manualmente, cortados em cubos de $20 \mathrm{~mm}$, pesados e então colocados em frascos de $600 \mathrm{~mL}$ com a solução desidratante nas concentrações de 40 a $60^{\circ}$ Brix, previamente aquecida em temperaturas de 30 ou $40^{\circ} \mathrm{C}$, de acordo com a Tabela 2 . A relação amostra:solução utilizada foi 1:10 (p/p), para garantir a concentração da solução constante durante todo o processo. Os ensaios de desidratação osmótica para a determinação da cinética do processo foram conduzidos 
à temperatura controlada em banho termostático, marca Tecnal, modelo TE-420 com agitação (120rpm) durante 8 horas com retirada de amostras nos tempos: $0,5,1,1,5$, 2, 3, 4, 6, 7 e 8 horas de processo.

TABELA 2 - Ensaios de cinética de desidratação osmótica

\begin{tabular}{cccc}
\hline Ensaio & $\begin{array}{c}\text { Tipo de } \\
\text { Açúcar }\end{array}$ & $\begin{array}{c}\text { Concentração } \\
\left({ }^{\circ} \text { Brix }\right)\end{array}$ & $\begin{array}{c}\text { Temperatura } \\
\left({ }^{\circ} \mathrm{C}\right)\end{array}$ \\
\hline 1 & Sacarose & 40 & 30 \\
2 & Sacarose & 50 & 30 \\
3 & Sacarose & 60 & 30 \\
4 & Sacarose & 40 & 40 \\
5 & Sacarose & 50 & 40 \\
6 & Sacarose & 60 & 40 \\
7 & Maltose & 40 & 30 \\
8 & Maltose & 50 & 30 \\
9 & Maltose & 60 & 30 \\
10 & Maltose & 40 & 40 \\
11 & Maltose & 50 & 40 \\
12 & Maltose & 60 & 40 \\
\hline
\end{tabular}

Em seguida, o melão foi drenado, enxagüado com água destilada para retirar o excesso de xarope, colocado sobre papel absorvente para a remoção da solução em excesso e pesado novamente. Amostras das frutas processadas osmoticamente foram submetidas às análises de umidade e açúcares totais [2] para a determinação das cinéticas de perda de água e ganho de sólidos, avaliados através das equações 1 e 2 .

$$
\begin{aligned}
& P A(\%)=100 \cdot\left[\frac{M_{0} \cdot X_{b u 0}-M_{f} \cdot X_{b u f}}{M_{0}}\right] \\
& G S(\%)=100 \cdot\left[\frac{A T_{,} \cdot M_{f}-A T_{0} \cdot M_{0}}{M_{0}}\right]
\end{aligned}
$$

onde:

PA $(\%)=$ perda de água percentual do produto desidratado;

$\mathrm{M}_{0}=$ massa inicial do produto;

$\mathrm{M}_{t}$ = massa final do produto;

$\mathrm{X}_{\mathrm{w} \mathrm{u}}=$ teor de umidade em base úmida inicial do produto;

$\mathrm{X}_{\mathrm{b}, \mathrm{l}}=$ teor de umidade em base úmida final do produto;

GS(\%) = incorporação de sólidos do produto desidratado;

$\mathrm{AT}_{\text {。 }}=$ teor de açúcares totais inicial do produto;

$\mathrm{AT}_{t}=$ teor de açúcares totais final do produto.

\section{3 - Determinação dos coeficientes de difusão da água e dos solutos}

A determinação dos coeficientes de difusividade da água e dos açúcares foi baseada no modelo proposto por AZUARA, BERISTAIN \& GARCIA [5] para a difusão em um cubo, em estado não estacionário. De acordo com os autores, partindo-se de um balanço de massa no material que sofrerá desidratação, obtém-se a seguinte equação para a perda de água (PA):

$$
P A=\frac{S_{1} \cdot t\left(P A_{\infty}\right)}{1+S_{1} t}
$$

onde:

$\mathrm{S}_{1}=$ constante relacionada à perda de água;

$\mathrm{PA}=$ perda de água no equilíbrio (\%).

Os valores da constante $\mathrm{S}$ e do termo PA (situação de equilíbrio) podem ser calculados a partir da linearização da equação 3:

$\frac{t}{P A}=\frac{1}{S_{1}\left(P A_{\infty}\right)}+\frac{t}{P A_{\infty}}$

Como as taxas de transferência de massa durante a desidratação osmótica são maiores no início do processo, é comum a utilização de versões simplificadas da equação de difusão de Fick, considerando um curto tempo de tratamento, regime transiente, difusão em um meio semi-infinito, concentração da solução osmótica constante e resistência externa à transferência de massa desprezível. Esta equação é apresentada por CRANK [6]:

$\frac{P A_{t}}{P A_{\infty}}=2 \cdot\left(\frac{D_{e f} t}{\pi L^{2}}\right)^{1 / 2}$

onde:

$\mathrm{PA}=$ perda de água no tempo $\mathrm{t}(\%)$;

$\mathrm{PA}_{v}=$ perda de água no equilíbrio (\%);

$\mathrm{D}_{\text {ct }}=$ difusividade efetiva $\left(\mathrm{m}^{2} / \mathrm{s}\right)$;

$\mathrm{L}=$ dimensão característica $(\mathrm{m})$.

A equação 5 indica que a quantidade de água saindo do alimento é diretamente proporcional à raiz quadrada do tempo, sendo que o valor de $\mathrm{D}_{\text {cr }}$ calculado corresponde a uma difusividade média para a faixa de concentrações durante o processo difusional.

Relacionando a equação 5 com o modelo proposto pela equação 3 , obtém-se uma expressão simples para calcular a difusividade da água a diferentes tempos:

$\left(D_{e f}\right)_{t}=\frac{\pi t}{4}\left[\left(\frac{S_{1} L}{1+S_{1} t}\right) \cdot\left(\frac{P A_{\infty}^{\mathrm{mod}}}{P A_{\infty}^{\mathrm{exp}}}\right)\right]^{2}$

onde:

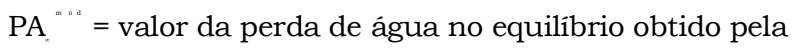
equação 4;

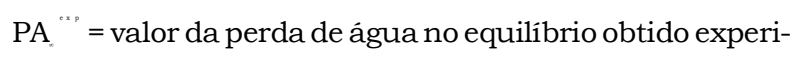
mentalmente;

$\left(D_{t}\right)=$ difusividade efetiva no tempot.

Quando PA * é desconhecido, assume-se que seu valor é igual a PA * e a equação 6 pode ser usada para obter boas estimativas para $\left(D_{c}\right)$, desde que os dados da cinética sejam adequadamente ajustados pela equação 3 .

Rearranjando a equação 6 para uma geometria cúbica, isto é, considerando a dimensão característica como 
sendo a aresta do cubo, e substituindo-a na equação 3, chega-se à equação 7 :

$D_{e f 1}=\frac{\pi}{4 t^{1 / 3}}\left[\left(\frac{S_{1} L^{3}}{1+S_{1} t}\right) \cdot\left(\frac{P A_{\infty}^{\bmod }}{P A_{\infty}^{\exp }}\right)\right]^{2 / 3}$

Analogamente, a difusividade dos solutos pode ser obtida pela equação 8:

$D_{e f 2}=\frac{\pi}{4 t^{1 / 3}}\left[\left(\frac{S_{2} L^{3}}{1+S_{2} t}\right) \cdot\left(\frac{G S_{\infty}^{\bmod }}{G S_{\infty}^{\exp }}\right)\right]^{2 / 3}$

A difusividade efetiva média $\overline{\mathrm{D}_{c}}$ foi calculada de acordo com a seguinte equação:

$\overline{D_{e f}}=\frac{\sum_{i=1}^{n}\left(D_{e f}\right)_{i}}{N}$

onde:

$\left(D_{c}\right)_{t}=$ difusividade efetiva no tempo $t$;

$\mathrm{N}=$ número de dados utilizados.

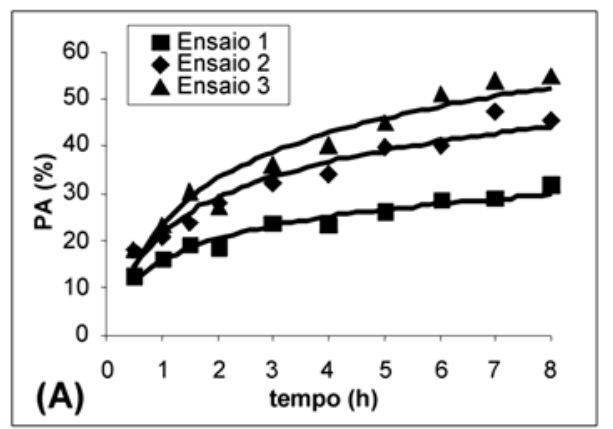

\section{3 - RESULTADOS E DISCUSSÃO}

Nas Figuras 1 a 4 são apresentadas as curvas referentes à perda de água e ganho de sólidos ao longo do processo nas 12 condições estudadas. As condições relativas aos ensaios realizados podem ser verificadas na Tabela 2 .

De acordo com as Figuras $1 \mathrm{~A}, 2 \mathrm{~A}, 3 \mathrm{~A}$ e $4 \mathrm{~A}$, verificouse uma maior taxa de saída de água da fruta nas duas primeiras horas de tratamento, principalmente nos ensaios realizados com maltose, conseqüência da grande força motriz existente entre a fruta e a solução hipertônica no início do processo. O aumento da concentração e da temperatura da solução favoreceram a perda de água no produto pelo maior gradiente de pressão osmótica na interface produto/solução, proporcionando uma maior taxa de transferência de massa. Comportamento semelhante foi observado por RASTOGI \& RAGHAVARAO [20], LAZARIDES, KATSANIDIS \& NICKOLAIDIS [13] e TALENS et al. [23] em trabalhos similares, onde os autores afirmam que maiores taxas de perda de água são obtidas com a combinação alta temperatura e concentração da solução hipertônica.

FIGURA 1 - (A) Cinéticas de perda de água (\%) e (B) Cinéticas de ganho de sólidos para os ensaios 1, 2 e 3 durante 8 horas de processo
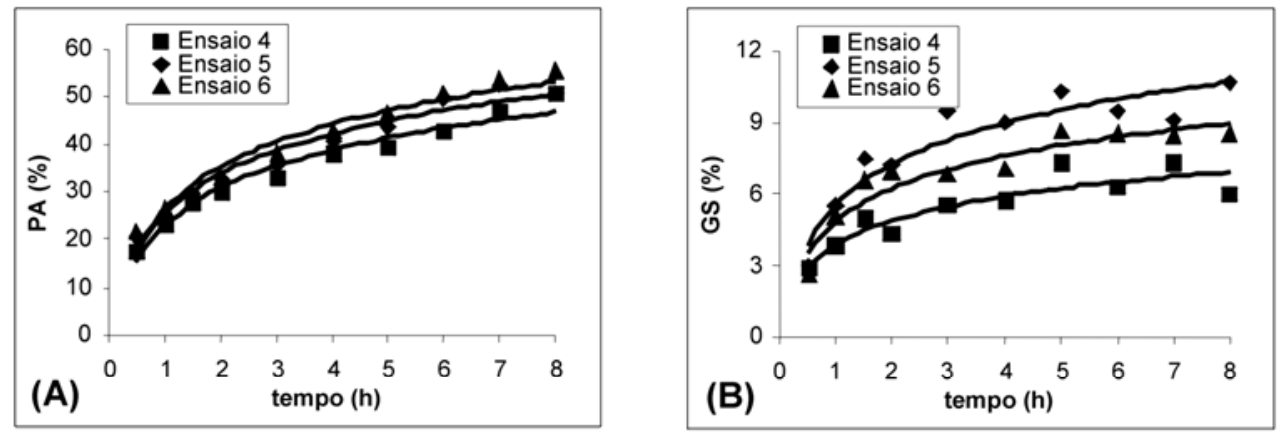

FIGURA 2 - (A) Cinéticas de perda de água (\%) e (B) Cinéticas de ganho de sólidos para os ensaios 4, 5 e 6 durante 8 horas de processo 

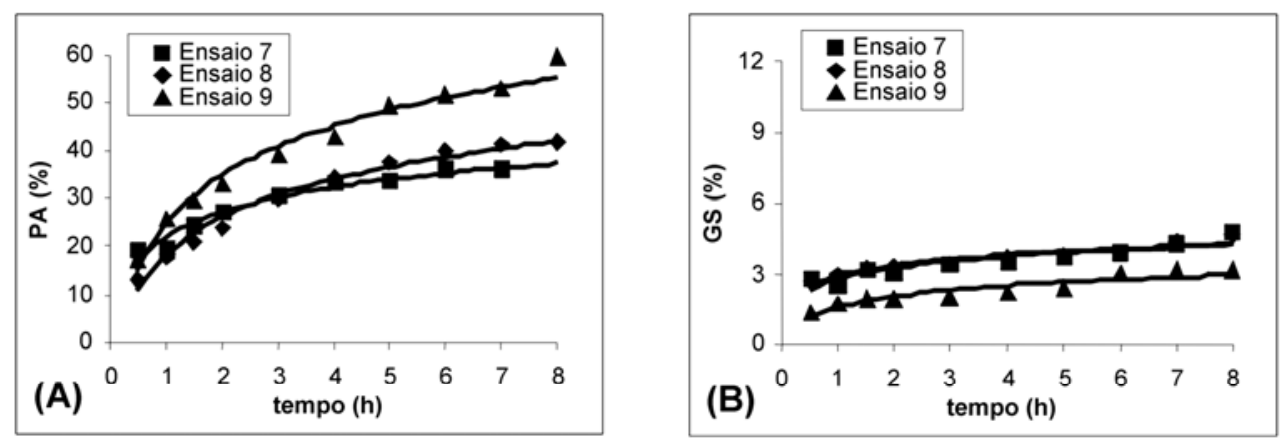

FIGURA 3 - (A) Cinéticas de perda de água (\%) e (B) Cinéticas de ganho de sólidos para os ensaios 7, 8 e 9 durante 8 horas de processo
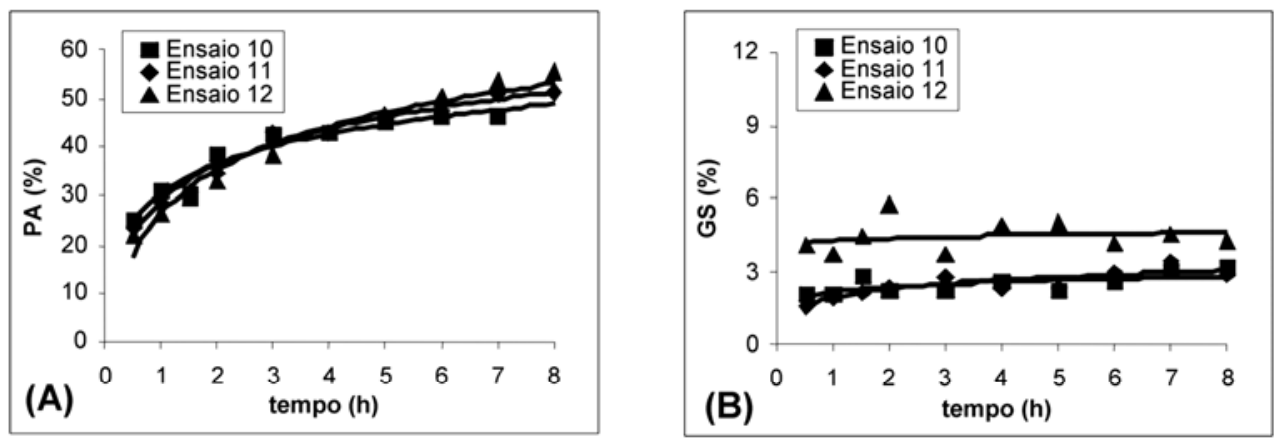

FIGURA 4 - (A) Cinéticas de perda de água (\%) e (B) Cinéticas de ganho de sólidos para os ensaios 10, 11 e 12 durante 8 horas de processo

Para o ganho de sólidos, foram observadas diferenças marcantes entre os tratamentos com sacarose e maltose (Figuras 1B, 2B, 3B e 4B). De maneira geral, o melão desidratado osmoticamente com solução de maltose apresentou uma incorporação de solutos inferior ao processado com sacarose ao longo do tempo, o que pode ser atribuído às diferenças entre o peso molecular das duas soluções utilizadas, fato que também foi verificado por LAZARIDES \& MAVROUDIS [12], LAZARIDES, KATSANIDIS \& NICKOLAIDIS [13] e FORNI et al. [9] nos seus trabalhos com batatas, maçãs e abricós, respectivamente.

Analisando o efeito da concentração da solução osmótica no ganho de sólidos, notou-se uma diminuição da incorporação tanto de sacarose quanto de maltose com o aumento da concentração da solução, pois soluções mais concentradas limitam a entrada de sólidos no produto pela formação de uma camada superficial de açúcar ao redor da fruta. SANJINEZ-ARGANDOÑA [22], TALENS et al. [23] e GIRALDO et al. [10] também observaram este comportamento em seus estudos com goiabas, kiwis e mangas, respectivamente. Entretanto, no presente trabalho, isso ocorreu apenas a temperatu- ra de $30^{\circ} \mathrm{C}$, quando a viscosidade da solução é maior, representando uma barreira para a transferência de massa da solução para a fruta. A redução da viscosidade da solução a $40^{\circ} \mathrm{C}$ resultou numa maior taxa de incorporação de sólidos, com o aumento da concentração da solução infusora.

Em relação à influência da temperatura, o melão desidratado osmoticamente com sacarose a $40^{\circ} \mathrm{C}$ (Figura $2 B)$ apresentou um ganho de açúcares superior ao processado a temperatura de $30^{\circ} \mathrm{C}$ (Figura $1 B$ ), favorecido pela temperatura mais alta que altera a permeabilidade da membrana celular, permitindo um maior ingresso de sacarose para a fruta, exceto para a concentração de $40^{\circ}$ Brix. Por outro lado, nos tratamentos com maltose, uma maior incorporação de açúcares foi verificada somente na concentração de $60^{\circ}$ Brix (Figura $4 B$ ), enquanto que nas outras concentrações ( 40 e $\left.50^{\circ} \mathrm{Brix}\right)$, observouse um menor ganho de sólidos a temperatura de $40^{\circ} \mathrm{C}$, fato que pode ser atribuido a uma possivel maior preservação da estrutura celular pela maltose, impedindo uma entrada elevada de açúcar para o interior da fruta numa temperatura mais alta. 
As difusividades efetivas médias $\overline{(\mathrm{D})}$ da água e dos solutos calculadas através do modelo de AZUARA, BERISTAIN \& GARCIA [5], assim como os respectivos coeficientes de regressão ( $\left.R^{2}\right)$ para todos os ensaios são apresentados na Tabela 3.

TABELA 3 - Difusividades efetivas médias para a água $\left(\overline{\mathrm{D}_{f^{\prime}}}\right)$ e solutos $(\overline{\mathrm{D}})$

\begin{tabular}{ccccc}
\hline Ensaio & $\overline{D_{f 1}}\left(10^{9} \mathrm{~m}^{2} / \mathrm{s}\right)$ & $\mathrm{R}^{2}$ & $\overline{D_{f 2}}\left(10^{9} \mathrm{~m}^{2} / \mathrm{s}\right)$ & $\mathrm{R}^{2}$ \\
\hline 1 & 6,891 & 0,977 & 5,214 & 0,948 \\
2 & 6,412 & 0,972 & 7,412 & 0,932 \\
3 & 6,296 & 0,974 & 3,647 & 0,922 \\
4 & 7,470 & 0,971 & 7,955 & 0,957 \\
5 & 6,657 & 0,979 & 8,516 & 0,976 \\
6 & 7,543 & 0,982 & 7,624 & 0,987 \\
7 & 6,961 & 0,993 & 11,348 & 0,967 \\
8 & 6,142 & 0,991 & 8,168 & 0,980 \\
9 & 6,120 & 0,979 & 6,627 & 0,939 \\
10 & 7,382 & 0,996 & 8,388 & 0,940 \\
11 & 7,443 & 0,993 & 8,018 & 0,942 \\
12 & 7,507 & 0,998 & 11,409 & 0,978 \\
\hline
\end{tabular}

Analisando a Tabela 3, é possivel afirmar que a temperatura apresentou uma influência mais significativa do que a concentração da solução na difusividade da água. Foi observado um aumento nos valores da difusividade com a elevação da temperatura, exceto para o tratamento com solução de sacarose a $50^{\circ}$ Brix que apresentou resultados muito semelhantes para a difusividade da água nas temperaturas de 30 e $40^{\circ} \mathrm{C}$. Esta ocorrência pode ser atribuída a alterações na estrutura celular da fruta, ocasionadas pela temperatura, resultando numa maior velocidade de difusão da sacarose para o interior da fruta em comparação com a difusão da água.

O aumento da concentração da solução desidratante apresentou efeitos distintos na difusividade da água, dependendo da temperatura de processo. Na temperatura de $30^{\circ} \mathrm{C}$, maiores concentrações de sacarose e maltose levaram a uma pequena diminuição dos coeficientes de difusividade da água, embora as taxas de perda de água tenham sido significativamente maiores nos ensaios utilizando maior concentração de solução osmótica (Figuras $1 \mathrm{~A}$ e $3 \mathrm{~A}$ ). Como a difusividade calculada se refere a uma média das taxas de difusão da água ao longo do processo, a tendência é a diminuição da velocidade de difusão com o tempo devido à aproximação do sistema ao equilíbrio, provocando uma redução dos coeficientes de difusão e, assim, menores valores para a difusividade média. TELIS, MURARI \& YAMASHITA [24] também verificaram um comportamento semelhante no estudo dos coeficientes de difusividade durante a cinética de desidratação osmótica de tomates. Por outro lado, a $40^{\circ} \mathrm{C}$, não se observou influência significativa da concentração de maltose e sacarose nas difusividades da água, com exceção do ensaio conduzido com sacarose na concentração de $50^{\circ}$ Brix, que resultou em um valor de difusividade efetiva inferior aos outros (Tabela 3), devido ao maior ganho de sólidos ocorrido nesta condição.

No caso dos solutos, tanto a concentração da solução como a temperatura apresentaram efeito significativo na difusão dos açúcares. A utilização da temperatura mais elevada $\left(40^{\circ} \mathrm{C}\right)$ na desidratação osmótica implicou em maiores valores das difusividades efetivas para a sacarose, provocados pela diminuição da viscosidade da solução, o que foi relatado por RASTOGI \& RAGHAVARAO [20] e KAYMAK-ERTEKIN \& SULTANOGLU [11]. No entanto, para a maltose este comportamento ocorreu apenas na concentração de $60^{\circ} \mathrm{Brix}$, enquanto que nas outras duas concentrações estudadas ( 40 e $50^{\circ} \mathrm{Brix}$ ) foi observado um decréscimo da difusividade efetiva do soluto com o aumento da temperatura devido ao menor ganho de açúcares ao longo do processo nestas condições. Por outro lado, a $30^{\circ} \mathrm{C}$, a difusividade dos açúcares tende a diminuir com o aumento da concentração da solução desidratante devido à formação da uma camada espessa de açúcar ao redor da fruta, dificultando a transferência de massa da solução para o produto, resultados que estão de acordo com os autores NSONZI \& RAMASWAMY [18] em trabalhos similares com mirtilo.

\section{4 - CONCLUSÕES}

Os resultados experimentais mostraram que o melão desidratado osmoticamente com solução de maltose apresentou simultaneamente, maiores taxas de perda de água e menor incorporação de sólidos ao longo do tempo em relação ao processado com sacarose, conseqüência das diferenças do peso molecular dos dois solutos.

O modelo empregado foi capaz de predizer a cinética de transferência de massa do processo osmótico de maneira satisfatória, apresentando coeficientes de difusão médios, da ordem de grandeza de $10^{\prime \prime}-10^{\circ} \mathrm{m}^{2} / \mathrm{s}$, tanto para a água quanto para os solutos, entretanto os dados experimentais de perda de água foram melhor ajustados pelo modelo, com valores do coeficiente de regressão ( $\left.R^{2}\right)$ superiores a 0,97.

\section{5 - REFERÊNCIAS BIBLIOGRÁFICAS}

[1] AGRIANUAL 2003. Anuário da Agricultura Brasileira. São Paulo: FNP - Consultoria e Comércio, 2002.

[2] A.O.A.C. (Association of Official Analytical Chemists) Official Methods of Analysis of the Association of Official Analytical Chemists, 16"' edition. Arlington: A.O.A.C., 1995.

[3] ARGAIZ, A.; LÓPEZ-MALO, A.; PALOU, E.; WELTI, J. Osmotic Dehydration of Papaya with Corn Syrup Solids. Drying Technology, v. 12, n. 7, p. 1709-1725, 1994.

[4] AZOUBEL, P.M.; MURR, F.E.X. Mathematical Modelling of the Osmotic Dehydration of Cherry Tomato (Lycopersicon esculentum var. cerasiforme). Ciênc. Tecnol. Aliment., v. 20, n. 2, p. 228-232, 2000.

[5] AZUARA, E.; BERISTAIN, C.I.; GARCIA, H.S. Development of a Mathematical Model to Predict Kinetics of Osmotic Dehydration. Journal of Food Science and Technology, v. 29, p. 239-242, 1992.

[6] CRANK, J. The Mathematics of Diffusion. $2^{\text {nd }}$ edition. Oxford: Clarendon, 1975.

[7] EL-AOUAR, A. A. Avaliação do processo combinado de desidratação osmótica e secagem na qualidade de cubos de mamão formosa (Carica papaya L.). 
Campinas, 2001, 113p. Dissertação (Mestre em Engenharia de Alimentos) - Faculdade de Engenharia de Alimentos, Universidade Estadual de Campinas.

[8] FITO, P.; CHIRALT, A.; BETORET, N.; GRAS, M.; CHÁFER, M.; MARTINÉZ-MONZÓ, J.; ANDRÉS, A.; VIDAL, D. Vacuum Impregnation and Osmotic Dehydration in Matrix Engineering. Application in Functional Fresh Food Development. Journal of Food Engineering, v. 49, p. 175-183, 2001.

[9] FORNI, E.; SORMANI, A.; SCALISE, S.; TORREGGIANI, D. The Influence of Sugar Composition on the Color Stability of Osmodehydrofrozen Moisture Apricots. Food Research International, v. 30, p. 87-94, 1997.

[10] GIRALDO, G.; TALENS, P.; FITO, B.; CHIRALT, A. Influence of Sucrose Solution Concentration on Kinetics and Yield during Osmotic Dehydration of Mango. Journal of Food Engineering, v. 58, p. 33-43, 2003.

[11] KAYMAK-ERTEKIN, F.; SUlTANOGLU, M. Modelling of Mass Transfer during Osmotic Dehydration of Apples. Journal of Food Engineering, v. 46, p. 243-250, 2000.

[12] LAZARIDES, H. N.; MAVROUDIS, N.E. Kinetics of Osmotic Dehydration of a Highly Shrinking Vegetable Tissue in a Salt-Free Medium. Journal of Food Engineering, v. 30, p. 61-74, 1996.

[13] LAZARIDES, H. N.; KATSANIDIS, E.; NICKOLAIDIS, A. Mass Transfer Kinetics during Osmotic Preconcentration Aiming at Minimal Solid Uptake. Journal of Food Engineering, v. 25, p. 151-166, 1995.

[14] LENART, A. Osmo-Convective Drying of Fruits and Vegetable: Technology and Application. Drying Technology, v. 14, n. 2, p. 391-413, 1996

[15] LIMA, A.S.; FIGUEIREDO, R. W.; MAIA, G.A.; LIMA, J.R.; SOUZA NETO, M. A.; SOUZA, A.C.R. Estudo das Variáveis de Processo sobre a Cinética de Desidratação Osmótica de Melão. Ciênc. Tecnol. Aliment., v. 24, n. 2, p. 282-286, 2004.

[16] MENDONÇA, F.V.S.; MENEZES, J.B.; GOIS, V.A.; GUIMARÃES, A.A.; NUNES, G.H.S.; MENDONÇA JUNIOR, C.F. Efeito do Retardamento da Colheita na Qualidade e na Vida Útil do Melão Orange Flesh. Horticultura Brasileira, v. 22, n. 1, p. 35-38, 2004.

[17] Menezes, J. B. Qualidade Pós-Colheita de Melão Tipo Galia Durante a Maturação e o Armazenamento. Lavras, 1996, 155p. Tese (Doutor em Ciência de Alimentos) - Departamento de Ciência dos Alimentos, Universidade Federal de Lavras.

[18] NSONZI, F.; RAMASWAMY, H.S. Osmotic Dehydration
Kinetics of Blueberries. Drying Technology, v. 16, p. 725-741, 1998.

[19] PARK, K.J.; BIN, A.; BROD, F.P.R.; PARK, T.H.K.B. Osmotic Dehydration Kinetics of Pear D'Anjou (Pyrus communis L.). Journal of Food Engineering, v. 52, p. 293-298, 2002.

[20] RASTOGI, N.K.; RAGHAVARAO, K.S.M.S. Mass Transfer during Osmotic Dehydration of Pineapple: considering Fickian Diffusion in Cubical Configuration. Lebensmittel-Wissenschaft und-Technologie, v. 37, p. 43-47, 2004

[21] RASTOGI, N.K.; RAGHAVARAO, K.S.M.S. Water and Solute Diffusion Coefficients of Carrot as a Function of Temperature and Concentration during Osmotic Dehydration. Journal of Food Engineering, v. 34, p. 429-440, 1997.

[22] SANJINEZ-ARGANDOÑA, E.J. Desidratação Osmótica de Goiaba por Imersão e Secagem. Campinas, 1999, 102p. Dissertação (Mestre em Engenharia de Alimentos) Faculdade de Engenharia de Alimentos, Universidade Estadual de Campinas.

[23] TALENS, P.; MARTÍNEZ-NAVARRETE, N.; FITO, P.; CHIRALT, A. Changes in Optical and Mechanical Properties during Osmodehydrofreezing of Kiwi Fruit. Innovative Food Science \& Emerging Technologies, v. 3, p. 191-199, 2002.

[24] TELIS, V.R.N.; MURARI, R.C.B.D.L.; YAMASHITA, F Diffusion Coefficients During Osmotic Dehydration of Tomatoes in Ternary Solutions. Journal of Food Engineering, v. 61, n. 2, p. 253-259, 2003.

[25] TORREgGiani, D.; BERTOLO, G. Osmotic PreTreatments in Fruit Processing: Chemical, Physical and Structural Effects. Journal of Food Engineering, v. 49, p. 247-253, 2001.

[26] WALISZEWSKI, K.N.; DELGADO, J.L.; GARCIA, M.A. Equilibrium Concentration and Water and Sucrose Diffusivity in Osmotic Dehydration of Pineapple Slabs. Drying Technology, v. 20, n. 2, p. 527-538, 2002.

\section{6 - AGRADECIMENTOS}

Os autores agradecem ao Conselho Nacional de Desenvolvimento Científico e Tecnológico (CNPq) e à Coordenadoria de Aperfeiçoamento de Pessoal de Nivel Superior (CAPES), pela concessão das bolsas e, à Fundação de Amparo à Pesquisa do Estado de São Paulo (FAPESP) pelo apoio financeiro. 